\title{
Hartree-Fock-Bogoliubov Calculations with Correlated Realistic Interactions
}

\author{
H. Hergert*, R. Roth, A. Zapp \\ Institut für Kernphysik, TU Darmstadt, Germany
}

May 21, 2007

\begin{abstract}
We investigate the use of correlated realistic interactions derived within the framework of the Unitary Correlation Operator Method (UCOM) in fully self-consistent Hartree-Fock-Bogoliubov calculations.
\end{abstract}

\section{Introduction}

Recently, we have successfully employed the Unitary Correlation Operator Method (UCOM) ([1] and Refs. therein) in nuclear structure studies using a wide range of many-body methods. It provides an efficient way to explicitly describe the strong central and tensor correlations which govern the nucleon-nucleon system at short ranges. By performing a similarity transformation of a given realistic $N N$-interaction with the unitary central and tensor correlation operators, $\mathrm{C}_{r}$ and $\mathrm{C}_{\Omega}$, we obtain a correlated realistic interaction $\mathrm{V}_{\mathrm{UCOM}}$ which retains phase-shift equivalence with its parent interaction while offering greatly improved convergence properties. The strength and range of the transformation are regulated by the central and tensor correlation functions $s(r)$ and $\vartheta(r)$, which are determined by an energy minimization in the two-nucleon system. For the free deuteron, this may lead to a very long-ranged $\vartheta(r)$, which is unnatural for many-nucleon systems where tensor correlations will be screened. Hence, we introduce a constraint $I_{\vartheta}^{(1,0)}$ on the volume integral (and thereby the range) of $\vartheta(r)$ in the $(S, T)=(1,0)$ channel. By varying this constraint, we were able, e.g., to map out the Tjon line $[1]$.

*E-Mail: Heiko.Hergert@physik.tu-darmstadt.de 

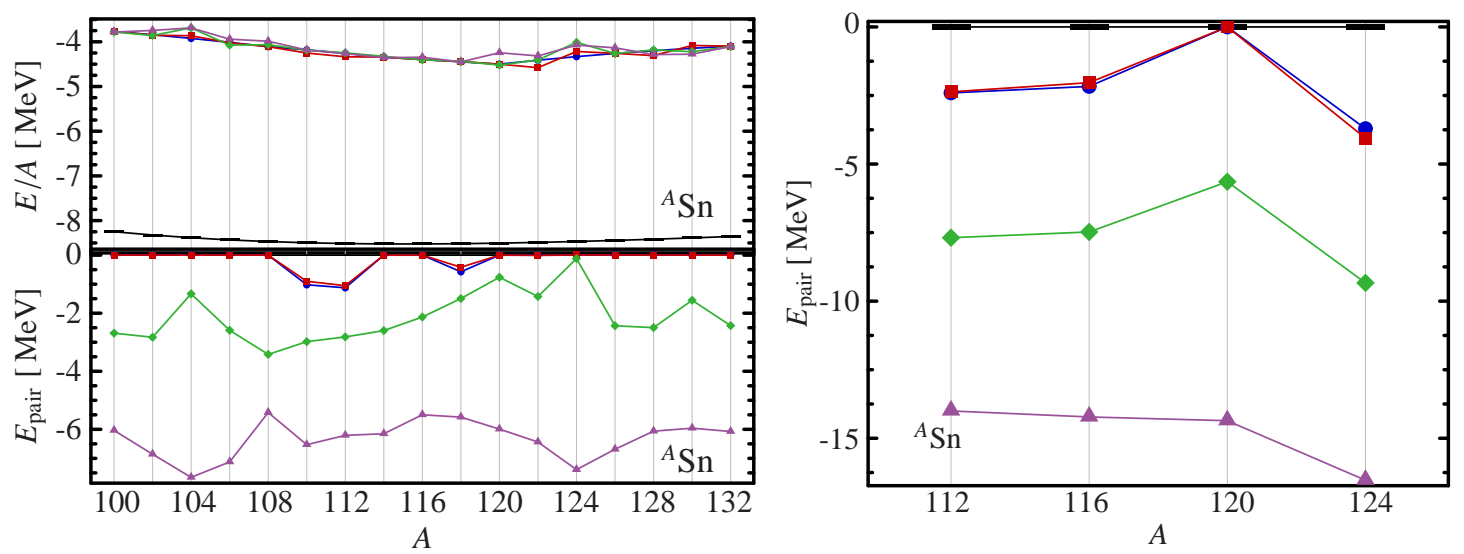

Figure 1: Left: Binding energy per nucleon and pairing energy for $\mathrm{V}_{\mathrm{UCOM}}$ with $I_{\vartheta}^{(1,0)}=0.09 \mathrm{fm}^{3}$. Right: Pairing energy for $\mathrm{V}_{\mathrm{UCOM}}$ with $I_{\vartheta}^{(1,0)}=0.2 \mathrm{fm}^{3}$ and a $3 N$ contact force with strength $V_{3 N}=2.5 \mathrm{GeV}$. In both figures, the shown curves are experiment (-), $\operatorname{HFB}(\bullet), \operatorname{PAV}(\boldsymbol{\square}), \operatorname{PLN}(\diamond), \operatorname{PM1}(\boldsymbol{\bullet})$.

\section{Hartree-Fock-Bogoliubov Calculations}

Hartree-Fock-Bogoliubov (HFB) theory [2] is the tool of choice for mean-field calculations including pairing effects. Starting from the nuclear many-body Hamiltonian, we use $\mathrm{V}_{\mathrm{UCOM}}$ in the particle-hole as well as the particle-particle channel. As in the Hartree-Fock (HF) calculations discussed in [3], we address the issue of the center-of-mass motion by using the intrinsic kinetic energy $\mathrm{T}_{\mathrm{int}}=\mathrm{T}-\mathrm{T}_{\mathrm{cm}}$ in the Hamiltonian rather than subtracting $\langle\mathrm{T}\rangle_{\mathrm{cm}}$ after the variation. Since $\mathrm{T}_{\mathrm{int}}$ is (formally) a two-body operator involving a summation over all relative kinetic energies of the nucleon pairs, it has to be included in the pairing field, along with the Coulomb force, to achieve a fully self-consistent treatment of direct, exchange, and pairing terms.

Proton and neutron numbers are restored simultaneously by using the LipkinNogami and $M$-periodic approximations $[4,5]$ to the full variation after projection (VAP) problem, which make use of truncated expansions of the number-projected energy. In addition, an exact particle-number projection (PNP) is performed after variation, as outlined in [6]. Curves in Fig. 1 are marked PLN and PM1, accordingly.

The left side of Fig. 1 shows results for the Sn isotope chain, calculated using a $\mathrm{V}_{\text {UCOM }}$ with $I_{\vartheta}^{(1,0)}=0.09 \mathrm{fm}^{3}$. Spherical and reflection symmetry were explicitly assumed. The binding energies agree with the HF result, and pairing is almost non-present. The reason for this is the low level density of the HF single-particle spectra, which impedes the pairing between different $(l, j)$-shells. As discussed in [7], the level density becomes comparable to those of single-particle spectra extracted from experiment if a $3 N$ contact force is used in addition to a $V_{U C O M}$ with a longer-ranged tensor correlator. We have tested this combination's effect on the pairing approximately by contracting the $3 N$ matrix elements with a den- 
sity matrix obtained from a HF run for a given nucleus and adding the resulting density-dependent $N N$ matrix elements to those of $\mathrm{V}_{\mathrm{UCOM}}$. This yields a more satisfying picture, as shown in the right panel of Fig. 1. As the sophistication of the approximate $\mathrm{PNP}$ increases $(\mathrm{PAV} \rightarrow \mathrm{PLN} \rightarrow \mathrm{PM} 1)$, we reach pairing energies up to $16 \mathrm{MeV}$, which are of comparable size, e.g, as the $E_{\text {pair }} \simeq 21-22 \mathrm{MeV}$ obtained from a VAP calculation with the Gogny D1 interaction [5]. Keeping in mind that the Gogny force has been adjusted to experimental data and therefore implicitly contains beyond mean-field effects, the trend noticed in the pairing energy as well as the remaining difference between the two approaches seem reasonable.

\section{Conclusions}

First results from fully self-consistent HFB calculations with a correlated realistic interaction $\mathrm{V}_{\mathrm{UCOM}}$ look promising and are in reasonable agreement with calculations based on established methods. Exact variation after PNP as well as a non-approximate treatment of the $3 N$ force are presently investigated. The reduction of symmetry constraints, which is likely to necessitate parity and/or angular momentum projection, will be a subject of future research. To improve the treatment of long-range correlations in our calculations we plan to develop a quasi-particle RPA code.

\section{Acknowledgment}

This work is supported by the Deutsche Forschungsgemeinschaft (DFG) under contract SFB 634.

\section{References}

[1] R. Roth et al., Phys. Rev. C 72 (2005) 034002

[2] P. Ring, P. Schuck, The Nuclear Many-Body Problem, 1st ed., Springer (1980)

[3] R. Roth et al., Phys. Rev. C 73 (2006) 044312

[4] H. Flocard and N. Onishi, Ann. Phys. (N.Y.) 254 (1997) 275

[5] M. Anguiano et al., Phys. Lett. B 545 (2002) 62

[6] J. A. Sheikh et al., Phys. Rev. C 66 (2002) 044318

[7] A. Zapp, diploma thesis (2006), TU Darmstadt 